\title{
Descrição morfológica dos ramos colaterais viscerais da aorta abdominal do macaco-de-cheiro
}

\author{
Morphological description of the collateral visceral branches from abdominal aorta of \\ squirrel monkey
}

\author{
Maria Rogéria Menezes da Silva ${ }^{\mathrm{I}}$ Ana Rita Lima ${ }^{\mathrm{I}}{ }^{*}$ Antônio Carlos Cunha Lacreta Junior ${ }^{1}$ \\ Mirian Naomi Ishizaki ${ }^{I}$ Aline Amaral ImbeloniI ${ }^{I}$ José Augusto Pereira Carneiro Muniz ${ }^{\text {II }}$ \\ Érika Renata Branco ${ }^{\mathrm{I}}$
}

\section{RESUMO}

O Saimiri sciureus é um primata não humano existente na região amazônica e não consta na lista de animais em perigo de extinção, de acordo com o IBAMA. Neste trabalho, sistematizou-se os ramos colaterais viscerais da aorta abdominal de seis animais sendo três machos e três fêmeas, os quais tiveram o sistema circulatório preenchido com látex Neoprene, adicionado de contraste radiográfico. O resultado observado foi que a aorta abdominal emitiu ventralmente, como ramo colateral visceral, a artéria celíaca, que se trifurcou nas artérias gástrica esquerda, hepática e lienal. Os ramos da artéria celíaca promovem a irrigação do estômago, duodeno, figado, pâncreas e baço. A seguir, a aorta abdominal emitiu a artéria mesentérica cranial, de calibre maior que a artéria celíaca e justaposta caudalmente. A artéria mesentérica cranial emitiu ramos que vascularizaram a parte final do duodeno, pâncreas, jejuno, íleo, ceco, cólon maior e cólon menor. A aorta abdominal emitiu lateralmente as artérias renais direita e esquerda. A artéria adrenal esquerda surgiu como um ramo colateral direto da artéria celíaca e a artéria adrenal direita surgiu da artéria renal direita. A artéria mesentérica caudal foi emitida da superfície ventral da aorta abdominal, logo abaixo das artérias renais. Em L6, a aorta abdominal se bifurcou, dando origem às artérias ilíacas externas direita e esquerda e estas deram origem à artéria ilíaca interna e femoral direita e esquerda. As artérias testiculares ou ováricas tiveram origem na artéria ilíaca interna. A continuidade da aorta abdominal deu origem à artéria sacral mediana $e$ a continuidade desta é chamada de artéria caudal mediana. $O$ estudo da espécie em questão é de suma importância para gerar conhecimentos a respeito dos primatas não humanos existentes em nosso país. Dessa forma, pode-se dizer que o Saimiri sciureus é um importante modelo biológico para o desenvolvimento de pesquisas biomédicas.

Palavras-chave: artérias, primatas, morfologia.

\begin{abstract}
The Saimiri sciureus is a non human primate that exists in the amazon region, which is not in the list of endangered animals according to IBAMA. So, this research systemized the collateral branches visceral of the abdominal aorta of these animals. It was used six animals, three males and three females, which had the circulatory system filled with latex Neoprene added of radiographic contrast. The results showed that the abdominal aorta ventrally emitted a collateral visceral branch called celiac artery, which originates three branches: left gastric, hepatic and lienal arteries. The branches of the celiac artery promoted the irrigation of the stomach, duodenum, liver, pancreas and spleen. Then the abdominal aorta emitted the cranial mesenteric artery, which was of a larger size than the celiac artery and was caudally juxtaposed. The cranial mesenteric artery supply branches that irrigate the final portion of the duodenum, pancreas, jejunum, ileum, cecum and colon. Abdominal aorta emitted laterally the right and left kidney arteries. The left adrenal artery as a collateral branch of the celiac artery and the right adrenal artery has been originated in the right renal artery. The mesenteric caudal artery was emitted from the ventral surface of the abdominal aorta, below the kidney arteries. In L6, abdominal aorta has two derivations to origin the right and left external iliac arteries and these had given origin to the internal iliac and femoral artery right and left. The testicular or ovary arteries were emitted in internal iliac arteries. Of the abdominal aorta led to origin the sacral median artery and in this sequence there is the caudal median artery. The study of this specie is very important to generate knowledge about non human primates existing in our country. It can be said that Saimiri sciureus is an important biological model for development of biomedical researches.
\end{abstract}

Key words: arteries, primates, morphology.

'Instituto de Saúde e Produção Animal (ISPA), Faculdade de Medicina Veterinária, Universidade Federal Rural da Amazônia (UFRA), Avenida Presidente Tancredo Neves, n.2501, Bairro Montese, 66077-530, Belém, PA, Brasil.E-mail: ana.lima@ufra.edu.br *Autor para correspondência.

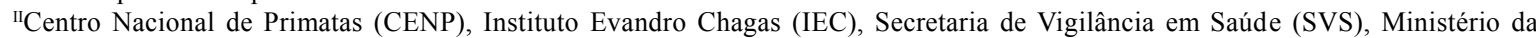
Saúde (MS), Ananindeua, PA, Brasil. 


\section{INTRODUÇÃO}

O território brasileiro possui a maior diversidade de primatas do mundo. São 120 espécies e subespécies. Desse total, 70\% estão na Amazônia, o que confere a essa região um verdadeiro atrativo para estudos da biodiversidade (DEL-CLARO \& FÁBIO, 2003). O estudo de primatas não humanos tem se tornado bastante importante para inúmeras pesquisas. Por outro lado, o conhecimento detalhado de sua anatomia pode representar um fator primordial para sua preservação, visto que tais informações podem ajudar na conservação das espécies (TEIXEIRA, 2005).

O Saimiri sciureus, conhecido como macaco-de-cheiro ou mão-de-ouro, habita em florestas primárias, secundárias, secas, tropicais de terra firme e nas temporariamente inundadas, em diferentes nichos ecológicos. Possui hábitos diurnos, sendo caracterizado por apresentar pelagem curta e espessa na cor verde-amarelada e extremidades amarelas; com cabeça arredondada e saliente na parte posterior, focinho curto e preto, com as orelhas arredondadas, dedos bem desenvolvidos com unhas planas, cauda não preênsil, dentes incisivos verticais e caninos grandes (AURICCHIO, 1995; COSTELLO et al., 1997). Essa espécie não faz parte da lista de animais ameaçados de extinção, de acordo com o IBAMA (2003).

Devido à falta de literatura disponível referente à ramificação da aorta abdominal em primatas, foram utilizadas informações de outros mamíferos sobre o assunto para a confecção da discussão. Foram encontradas descrições a esse respeito no ratão do banhado (Myocastor coypus) (CULAU et al., 2008), suínos, coelhos, cães e ovinos (EVANS \& DE LAHUNTA, 1994; GHOSHAL, 1986; KÖNIG \& LIEBICH, 2004), em cutias (CARVALHO et al., 1999) e no ouriço-cacheiro (Sphiggurus spp.) (MACHADO \& ZIEMMAN, 2000).

Este trabalho teve como objetivo sistematizar e descrever os ramos colaterais viscerais da aorta abdominal em Saimiri sciureus.

\section{MATERIAL E MÉTODO}

Foram estudados seis exemplares de Saimiri sciureus, sendo três machos e três fêmeas, que vieram a óbito por causas naturais, provenientes do Centro Nacional de Primatas - CENP, Ananindeua - PA, aprovado em Comitê de ética, Registro CEPAN - 008/ 2010 (Comitê de Ética em Pesquisa Animal do Instituto Evandro Chagas- Ananindeua- PA).

Os animais foram dissecados em nível de arco aórtico, através do $5^{\circ}$ espaço intercostal esquerdo, o qual foi canulado e injetado solução de látex Neoprene 650 , corado com pigmento vermelho e adicionado de contraste baritado para visualização dos vasos por meio de radiografia. Em seguida, os animais foram fixados e conservados com solução aquosa de formol a $10 \%$ por um período mínimo de $48 \mathrm{~h}$. Decorrido esse período, os animais foram radiografados. O equipamento utilizado nessa técnica é da marca Intecal ${ }^{\circledR}$, modelo CR-7 e potência de $100 \mathrm{kV}$ e $100 \mathrm{~mA}$. Foram utilizados filmes de $24 \times 30 \mathrm{~cm}$ da marca $A G F A{ }^{\circledR}$, mantendo uma distância de $90 \mathrm{~cm}$ entre o foco e o filme, com uma exposição de $50 \mathrm{kV}, 100 \mathrm{~mA}$ e tempo 0,2 segundos e as radiografias foram processadas manualmente. Esse método foi utilizado para complementar os resultados obtidos com a dissecação e algumas das ramificações da aorta abdominal não foram visualizadas por meio dessa técnica devido à sobreposição dessas ramificações. Após a análise radiográfica, os animais foram dissecados para a exposição das artérias em estudo. Para isso, foi feita uma incisão na cavidade abdominal, permitindo acesso aos ramos da aorta, os quais foram cuidadosamente dissecados e confeccionados desenhos esquemáticos, representativos da localização e origem dos ramos colaterais da aorta abdominal.

Toda nomenclatura adotada foi baseada na Nomina Anatomica Veterinaria (INTERNATIONAL COMMITTEE ON VETERINARY GROSS ANATOMICAL NOMENCLATURE, 2005).

\section{RESULTADOS}

A aorta abdominal do $\boldsymbol{S}$. sciureus apresentou origem em T12, emergindo na cavidade abdominal após atravessar o hiato aórtico entre os pilares do diafragma, acompanhando o teto do abdome e relacionando-se com a veia cava caudal à sua direita e com os músculos psoas à sua esquerda.

A primeira ramificação que surge na aorta abdominal (Figuras 1A e B, 2A e B) é a artéria celíaca (Figuras 1B e 2B) que, por sua vez, emite três ramos (artéria hepática, artéria gástrica esquerda e artéria lienal) (Figuras 1B e 2A). A artéria gástrica esquerda vasculariza o fundo e a região cárdica do estômago e emite ramos esofágicos, sendo a região pilórica vascularizada pela artéria gástrica direita. A artéria hepática dirige-se para o fígado e emite três ramos colaterais: os ramos pancreáticos, a artéria gástrica direita e a gastroduodenal. Essa última se divide em artéria gastrepiplóica direita e pancreaticoduodenal cranial. A artéria lienal, o maior ramo da artéria celíaca, emite quatro ramos que vão irrigar o pâncreas (ramos pancreáticos), o baço (ramos esplênicos) e o estômago 

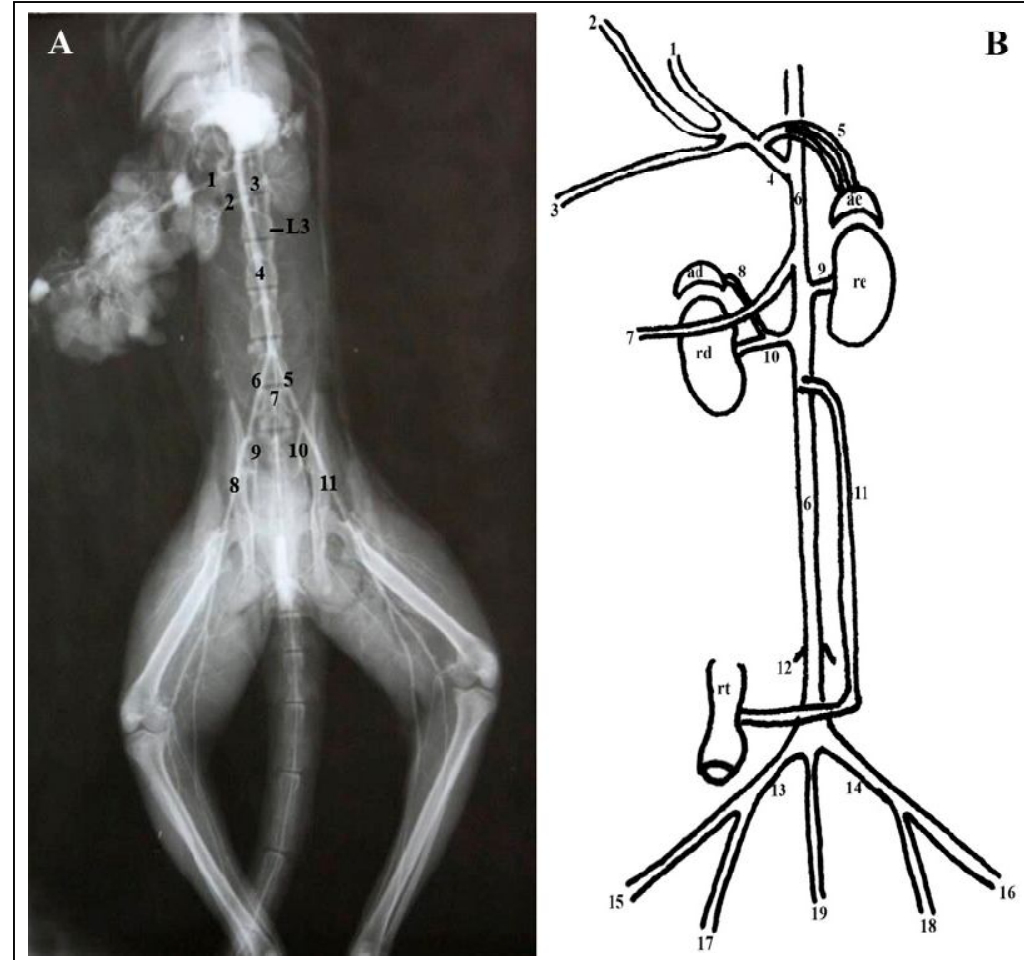

Figura 1 - A - Imagem radiográfica evidenciando os ramos da aorta abdominal do $\boldsymbol{S}$. sciureus em que se observam as artérias mesentérica cranial (1), renal direita (2) e esquerda (3), aorta abdominal (4), ilíaca externa esquerda (5) e direita (6), sacral mediana (7), femoral direita (8), ilíaca interna direita (9) e esquerda (10) e femoral esquerda (11), terceira vértebra lombar (L3). B - Desenho esquemático em vista ventral da aorta abdominal e seus principais ramos arteriais colaterais e terminais: gástrica esquerda (1), hepática (2), lienal (3), celíaca (4), adrenal esquerda (5), aorta abdominal (6), mesentérica cranial (7), adrenal direita (8), renal esquerda (9), renal direita (10), mesentérica caudal (11), testicular ou ovárica (12), ilíaca externa direita (13) e esquerda (14), femoral direita (15) e esquerda (16), ilíaca interna direita (17) e esquerda (18), sacral mediana (19), glândula adrenal esquerda (ae), glândula adrenal direita (ad), rim esquerdo (re), rim direito (rd) e reto (rt).

(artérias gástricas curtas e artéria gastrepiplóica esquerda). A artéria gastroepiplóica esquerda avança para a direita terminando na artéria gastroepiplóica direita. A artéria adrenal esquerda tem origem na artéria celíaca e a artéria adrenal direita tem origem na artéria renal direita.

A artéria mesentérica cranial (Figuras 1A, 1B, 2A, B e C) aparece como a segunda ramificação da aorta, surgindo ao nível de L2 na face ventral da aorta abdominal. Trata-se de um grande vaso ímpar que passa ventralmente pela veia cava caudal e penetra no mesentério. As artérias renais direita e esquerda (Figuras 1A e B, 2A) tiveram origem entre L2 e L3. A artéria mesentérica caudal (Figuras 1B, 2A e C) originouse em L3 na face ventral da aorta abdominal, apresentando uma extensão muito maior do que a artéria mesentérica cranial e suprindo a maior parte do cólon maior e o reto.

As artérias ilíacas externas surgem da bifurcação da aorta ao nível de L6. As artérias ilíacas externas direita e esquerda (Figuras $1 \mathrm{~A}$ e B, 2C e 3B) dão origem às artérias ilíacas internas direita e esquerda (Figuras 1A e B, 2C e 3B) e respectivas artérias femorais (Figuras $1 \mathrm{~A}$ e $\mathrm{B}$ e 2C). As artérias testiculares ou ováricas (Figuras 1B, 3B e C) têm origem na face medial das artérias ilíacas internas. As artérias ováricas são longas e delgadas, direcionando-se cranialmente para irrigar os órgãos-alvos (tubas uterinas e ovários). As artérias testiculares são curtas e delgadas e se direcionam medialmente para irrigar os testículos, epidídimos e ductos deferentes. A continuidade da aorta abdominal após a bifurcação em artérias ilíacas externas dá origem à artéria sacral mediana (Figuras $1 \mathrm{~A} \mathrm{e} \mathrm{B,} \mathrm{2C} \mathrm{e} \mathrm{3B).}$ 


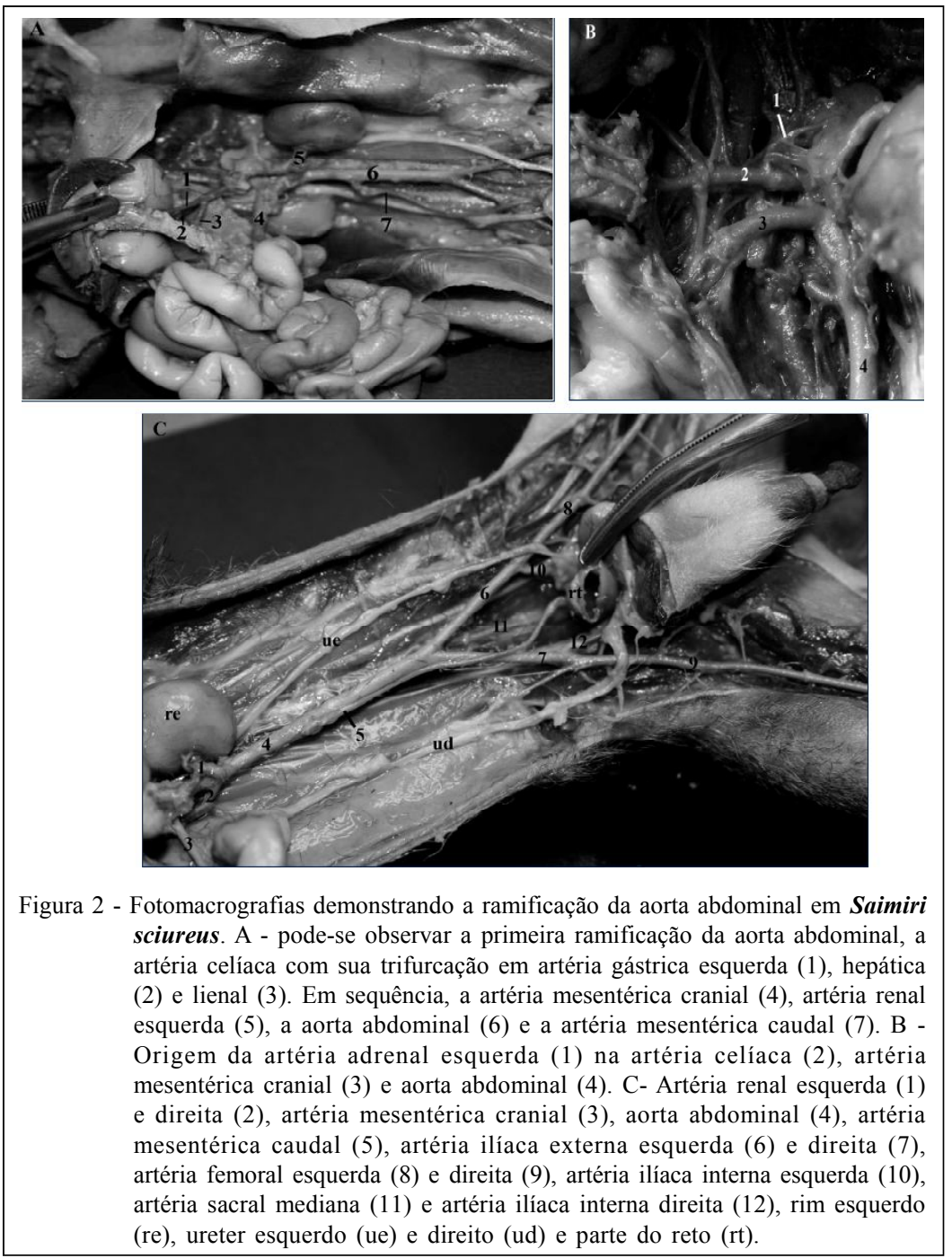

\section{DISCUSSÃO}

Existem relatos quanto à origem da artéria celíaca no ratão do banhado, que pode ter origem comum com a artéria mesentérica cranial ou separada desta (MACHADO et al., 2002a). Essas artérias originam-se independentemente uma da outra em suínos, coelhos e cães, mas por tronco comum em ovinos (GHOSHAL, 1986; KÖNIG \& LIEBICH, 2004). Neste trabalho, não ocorreu, em nenhum caso, a formação de um tronco comum entre as artérias celíaca e mesentérica cranial. A artéria celíaca do Saimiri sciureus é o primeiro ramo visceral, no sentido crâniocaudal. É curta, de grande calibre e apresenta três ramos com mesma origem: a artéria hepática, a artéria gástrica esquerda e a artéria lienal e, da mesma forma, EVANS \& DE LAHUNTA (1994) a descreveram no cão.
Nesta pesquisa, a artéria adrenal esquerda tem origem na artéria celíaca e a artéria adrenal direita origina-se da artéria renal direita, diferindo dos relatos da literatura. No ratão-do-banhado, as artérias adrenais recebem vascularização distinta para o lado direito e esquerdo. Para a glândula adrenal direita, destinam-se ramos provenientes das artérias frênica caudal direita, abdominal cranial direita, renal direita, primeira artéria lombar e aorta abdominal. Já para a glândula adrenal esquerda, existem ramos oriundos das artérias renal esquerda, aorta abdominal, abdominal cranial esquerda e segunda artéria lombar (MACHADO et al., 2002a). No cão, as artérias adrenais têm origem em locais diferentes e de maneira variável. Os ramos adrenais, cranial e caudal, normalmente surgem da artéria frênicoabdominal ou da artéria frênico caudal e da artéria renal, respectivamente. Entretanto, elas podem originar- 


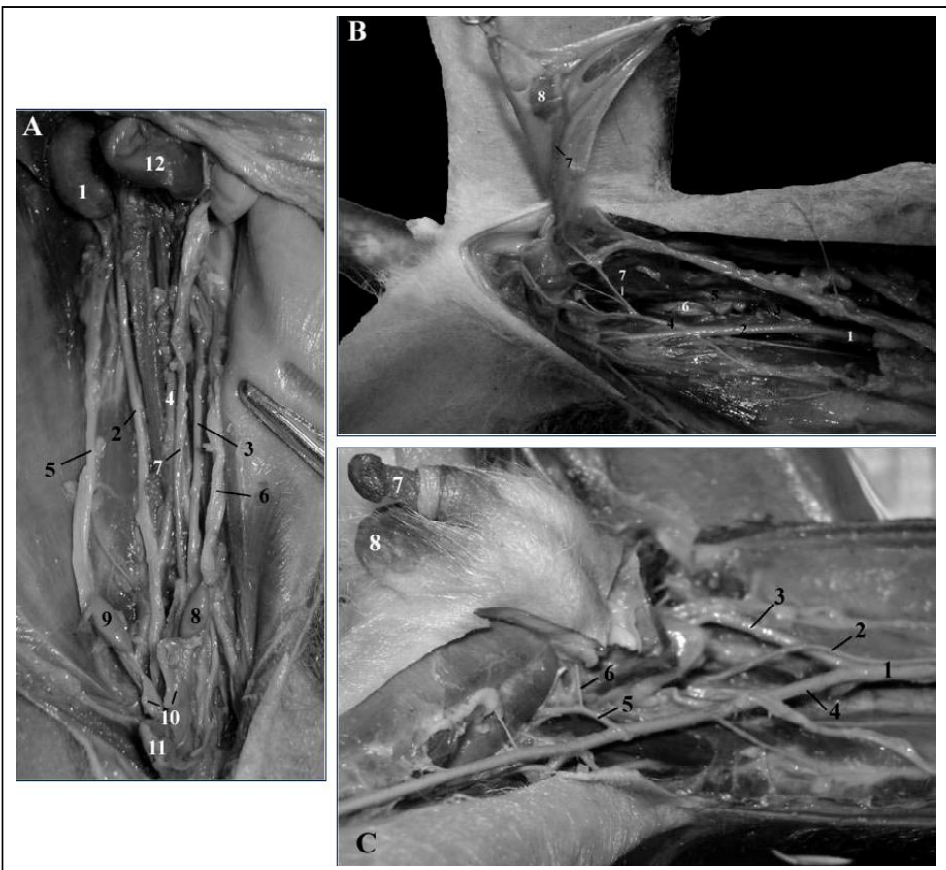

Figura 3 - A - Topografia visceral de um exemplar do sexo feminino de Saimiri sciureus, em que se pode observar o rim direito (1) o ureter direito (2) e esquerdo (3), aorta abdominal (4), mesovário direito (5) e esquerdo (6), artéria mesentérica caudal (7), ovário esquerdo (8) e direito (9), tubas uterinas (10), bexiga urinária (11) e jejuno (12). B - Fotomacrografia demonstrando os ramos terminais da aorta abdominal (1) em Saimiri sciureus, em que se pode observar a artéria ilíaca externa esquerda (2) e direita (3), artéria ilíaca interna esquerda (4) e direita (5), artéria sacral mediana (6), a artéria ovárica esquerda (7) e o ovário esquerdo (8). C- Topografia do sistema reprodutor masculino do Saimiri sciureus, em que se pode observar a aorta abdominal (1), a artéria mesentérica caudal (2), a artéria ilíaca externa direita (3) e esquerda (4), a artéria ilíaca interna esquerda (5), a artéria testicular esquerda (6), o pênis (7) e o escroto (8).

se da artéria lombar, da artéria celíaca e da mesentérica cranial. A artéria adrenal média, a principal supridora da glândula, tem origem na aorta abdominal ou na artéria renal (GETTY, 1986; DONE et al., 2002).

A artéria mesentérica cranial foi o vaso de maior calibre no $\boldsymbol{S}$. sciureus e tem origem próxima e caudalmente à artéria celíaca, na superfície ventral da aorta abdominal. Segue em direção ao mesentério, onde origina seus ramos para suprir principalmente a metade caudal do duodeno, até a parte cranial do cólon descendente (GHOSHAL, 1986). Quanto a sua origem, ventral à aorta, a artéria mesentérica cranial foi relatada nos animais domésticos, no rato de laboratório, no ouriço-cacheiro (Sphiggurus spp.) e no ratão-dobanhado (Myocastor coypus) (GHOSHAL, 1986; HEBEL \& STROMBERG, 1986; MACHADO \& ZIEMMAN, 2000; MACHADO, et al., 2006).

Caudalmente à origem da artéria mesentérica cranial, a aorta abdominal emitiu lateralmente as artérias renais direita e esquerda, que se tratam de vasos calibrosos no $\boldsymbol{S}$. sciureus. A artéria renal direita surge ligeiramente mais cranial que a esquerda, em conformidade com a posição mais cranial do rim direito, assemelhando-se ao que ocorre nos cães (GHOSHAL, 1986).

Em cutias adultas, a artéria mesentérica caudal bifurca em artéria cólica esquerda e artéria retal cranial (CARVALHO et al., 1999). No cão, a artéria mesentérica caudal, ímpar, é pequena e surge ventralmente da aorta abdominal. Supre as partes média e caudal do cólon descendente e a parte cranial do reto. Após percurso de cerca de cinco centímetros, ela se divide nas artérias cólica esquerda e retal cranial (GHOSHAL, 1986). Essa disposição da artéria mesentérica caudal foi a mesma encontrada no Saimiri sciureus, somente sua origem mais cranial e seu comprimento mais longo a torna diferente das outras espécies. 
No $\boldsymbol{S}$. sciureus, as artérias testiculares ou ováricas apresentaram localização diferenciada do descrito na literatura para as espécies domésticas (GHOSHAL, 1986) e para o macaco Rhesus (GINTHER et al., 1974), tendo origem nas artérias ilíacas internas, sendo que as artérias ováricas se dirigiram cranialmente e as testiculares dirigiram-se medialmente. Essa característica diferiu nas espécies citadas, nas quais as artérias testiculares ou ováricas são vasos pequenos, emitidos ventrolateralmente da aorta abdominal, aproximadamente a meio caminho entre as artérias renais e mesentérica caudal. Elas apresentaram origem cranial, seguindo em sentido caudal para irrigar os ovários e tubas uterinas ou, no caso dos machos, os testículos, epidídimos e os ductos deferentes.

A artéria ovárica no $\boldsymbol{S}$. sciureus apresentouse tortuosa como na vaca e na ovelha (DEL CAMPO \& GINTHER, 1973), sendo relativamente reta em equinos (GINTHER \& BISGARD, 1972) e suínos (DEL CAMPO \& GINTHER, 1973). Com relação à topografia dos órgãos reprodutores femininos do $\boldsymbol{S}$. sciureus, ela difere das espécies domésticas, pois apresenta os ovários localizados na parte caudal da cavidade abdominal, semelhante à espécie humana (VALERIUS, 2009), enquanto que, nas espécies domésticas, esses órgãos se encontram na cavidade abdominal, próximos aos rins (EVANS \& DE LAHUNTA, 1994).

A artéria testicular no $\boldsymbol{S}$. sciureus apresentou trajeto curto e direcionado medialmente, diferentemente do encontrado para as espécies domésticas, as quais são longas e delgadas e se originam entre as artérias renais e a mesentérica caudal (GHOSHAL, 1986).

Os ramos terminais da aorta abdominal foram as artérias ilíacas externas e a sacral mediana. A artéria ilíaca externa bifurca-se, dando origem à artéria ilíaca interna e à artéria femoral em ambos os lados. A artéria sacral mediana trata-se do ramo terminal da aorta abdominal. Essa conformação difere dos relatos em carnívoros, onde a artéria ilíaca externa surge como ramo na face lateral da aorta abdominal. Também é diferente dos ruminantes, nos quais a aorta se bifurca na artéria ilíaca externa e interna e a sacral mediana aparece como ramo da interna (GHOSHAL, 1986). No gambá, a aorta abdominal termina como artérias ilíacas comuns, as quais emitem as artérias ilíacas interna e externa como ramos. Já, no rato, as artérias ilíacas comuns lançam a artéria ilíaca interna e continuam como artéria ilíaca externa, fato também observado na nutria (Myocastor coypus), sendo que a artéria sacral mediana, nesses animais, é ramo da face dorsal da aorta abdominal, antes da divisão terminal (CULAU et al., 2008).

\section{CONCLUSÃO}

Os ramos colaterais do Saimiri sciureus emitidos direto da aorta abdominal são as artérias viscerais: celíaca, mesentérica cranial, renais e mesentérica caudal. Os ramos terminais são as artérias ilíaca externa, a sacral mediana e a caudal mediana. As testiculares ou ováricas têm origem nas artérias ilíacas internas.

\section{REFERENCIAS}

AURICCHIO, P. Primatas do Brasil. Guarulhos: Terra Brasilis, 1995. 168p.

CARVALHO M.A.M. et al. Artérias mesentéricas cranial e caudal em cutias (Dasyprocta aguti). Veterinária Notícias, v.2, p.17-24, 1999.

COSTELlO, R.K. et al. Squirrel monkeys (genus Saimiri) taxonomy: a multidisciplinary study of the biology of the species. In: KIMBEL, W.H.; MARTIN, L.B. Species, species concepts, and primate evolution. New York: Plenum, 1997. p. 177-210.

CULAU, P.O.V. et al. Ramos colaterais parietais e terminais da aorta abdominal em Myocastor coypus (nutria). Ciência Rural, v.38, n.4, p.997-1002, 2008. Disponível em: <http:// www.lume.ufrgs.br/bitstream/handle/10183/21984/ 000658384.pdf? sequence=1>. Acesso em: 20 abr. 2010. doi: 10.1590/S0103-84782008000400014.

DEL CAMPO, C.H.; GINTHER, O.J. Vascular anatomy of the uterus and ovaries and the unilateral luteolytic effect of the uterus: horses, sheep, and swine. American International Veterinary Research, v.34, p.305, 1973. Disponível em: $<$ http://www.ncbi.nlm.nih.gov/pubmed/4570830>. Acesso em: 20 abr. 2010 .

DEL-CLARO, K.; FÁBIO, P. As distintas faces do comportamento animal. Jundiaí: Sociedade Brasileira de Etologia, 2003. 276p.

DONE, S.H. et al. Atlas colorido de anatomia veterinária. Barueri: Manole, 2002. 275p.

EVANS, H.E.; LAHUNTA, A. Abdome, pelve e membro pélvico. In: __ Guia para a dissecação do cão. 3.ed. Ithaca: Edwards Brothers, 1994. p.118-119.

GETTY, R. Anatomia dos animais domésticos. 5.ed. Rio de Janeiro: Guanabara Koogan, 1986. 2v.

GINTHER, O.J. et al. Anatomy of arteries and veins of uterus and ovaries in Rhesus monkeys. Biology of Reproduction, v.11, p.205-219, 1974. Disponível em: <http://www.ncbi.nlm.nih.gov/ pubmed $>$. Acesso em: 15 abr. 2010.

GINTHER, O.J.; BISGARD, G.E. Role of the main uterine vein in local action of an intrauterine device on the corpus luteum in sheep. American International Veterinary Research, v.33, p.1583, 1972. Disponível em: <http://www.ncbi.nlm.nih.gov/ pubmed $>$. Acesso em: 15 abr. 2010. 
GHOSHAL, N.G. Coração e artérias do carnívoro. In: GETTY, R. Sisson/Grossman anatomia dos animais domésticos. Rio de Janeiro: Guanabara Koogan, 1986. p.1497-1550.

HEBEL, R.; STROMBERG, M.W. Anatomy and embryology of the laboratory rat. München: Bio Med Verlag Wörthsee, 1986. 270p.

IBAMA. Lista da fauna brasileira ameaçada de extinção, 2003. Online. Disponível em: <http://www.ibama.gov.br/fauna/ extincao.php>. Acesso em: 10 jun. 2010

INTERNATIONAL COMMITTEE ON VETERINARY GROSS ANATOMICAL NOMENCLATURE. Nomina anatomica veterinaria. 5.ed. Hannover, Columbia, Gent, Sapporo: Editorial Committee, 2005. 166p.

KÖNIG, H.E.; LIEBICH, H.G. Anatomia dos animais domésticos, texto e atlas colorido. Órgãos e sistemas. Porto Alegre: Artmed, 2004. V.2, p.69.

MACHADO, G.V. et al. Padrão de divisão e distribuição das artérias mesentéricas no ratão-do-banhado (Myocastor coypus - Rodentia: Mammalia). Biotemas, v.1, p.59-63, 2006. Disponível em: <http://www.biotemas.ufsc.br/volumes/pdf/ volume191/p59a63.pdf $>$. Acesso em: 20 abr. 2010. ISSN: $0103-1643$.
MACHADO, G.V. et al. Suprimento arterial para as glândulas adrenais no ratão-do-banhado (Myocastor coypus Molina, 1782). Archives of Veterinary Science, v.2, p.9-14, 2002a. Disponível em: <http://www. ojs.c3sl.ufpr.br/ojs2/index.php/ veterinary/article/download/ 3976/3216>. Acesso em: 17 abr. 2010. ISSN: 1517-784X.

MACHADO, G.V. et al. A artéria celíaca e seus ramos no ratão-do banhado (Myocastor coypus - Rodentia: Mammalia). Biotemas. v.2, p.41-54, 2002b. Disponível em: <http://www.biotemas.ufsc.br/ volumes/pdf/restaurados/15 2/41-54.pdf $>$. Acesso em: 17 abr. 2010 .

MACHADO, G.V.; ZIEMMAN, F.P. Comportamento anatômico das artérias mesentéricas no ouriço-cacheiro (Sphiggurus spp. Cuvier, 1825). In: EVINCI - EVENTO DE INICIAÇÃO CIENTÍFICA DA UFPR, 8., 2000, Curitiba, PR. Anais.... Curitiba: UFPR, 2000. p.250.

TEIXEIRA, D.G. Estudo anatômico descritivo dos órgãos genitais masculinos do macaco-prego (Cebus apella Linnaeus, 1758). 2005. 191f. Tese (Doutorado em Ciências) - Universidade de São Paulo, SP.

VALERIUS, K.P. Atlas de anatomia. São Paulo: Grupo Editorial Nacional, 2009. 592p. 